\title{
Mathematical Analysis of the Rolling Bearing Vibration
}

\section{Corrugated Surface}

\author{
Ding Wei ${ }^{1, a^{*}}$ \\ ${ }^{1}$ The School of Applied Technology, University of Science and Technology Liaoning, \\ Anshan Liaoning 114000, China \\ aabc5851547@sina.com
}

Keywords: Rolling bearing, Vibration, Mathematical analysis

Abstract. In order to study the surface ripples caused by outer ring vibration, we took the outer ring raceway and rolling body as the research object of the contact. The surface corrugation of the inner ring or outer ring would cause a periodic change of the distance between the inner and outer ring raceway. The surface corrugation of the roller could cause periodic change of roller diameter. Under the condition of the bearing no clearance operation, when rolling body made by operation, the changes would inevitably cause the radial displacement of the contact of the raceway and rolling body. The balance of outer ring raceway position changed. The radial displacement total of the outer ring rolling path with all the contact points was the outer radial vibration.

\section{Introduction}

With the development of science and technology the vibration and noise of rolling bearings are more and more concerned by people. The bearing vibration and noise becomes an important measure of evaluation quality. Result in rolling bearing vibration can be caused by many factors. Bearing inner and outer ring raceway and the surface of the corrugated roller are important reasons.

The influence of the harmonic lines acting on the contact points of outer orbits radial displacement

If the surface corrugations of inside and outside the circle orbit and rolling body were harmonic lines we could get

$$
\Delta R=A \cos \left(\mathrm{n} \varphi+\varphi_{0}\right)
$$

Where: $\Delta R$ is the surface undulation of the inside and outside the circle orbit or rolling body. Embossment is plus and hollow is minus, $\varphi$ is the position angle of any point relative to the ripple origin, $A$ is the amplitude of the surface corrugations, $\varphi_{0}$ is the initial phase angle of the surface corrugations, $\mathrm{n}$ is the number of waves of the inside and outside the circle orbit and the roller circumferential direction. We took any roller $(j)$ as the research object, as shown in Fig.1. The initial position angle was $\varphi_{j}$. The instant needle position angle was $\theta_{j}$. Clockwise rotation was plus. We could get

$$
\varphi_{j}=\frac{2 \pi}{Z}(j-1)+\varphi_{1}, j=1,2, \ldots, Z
$$




$$
\theta_{\mathrm{j}}=\frac{2 \pi}{\mathrm{Z}}(\mathrm{j}-1)+\varphi_{1}-\omega_{C} t
$$

Where: $Z$ is the number of the roller; $\varphi_{1}$ is the initial position angle of the roller $1 ; \omega_{c}$ is the orbital angular frequency of the roller; $I$ is the bearing running time.

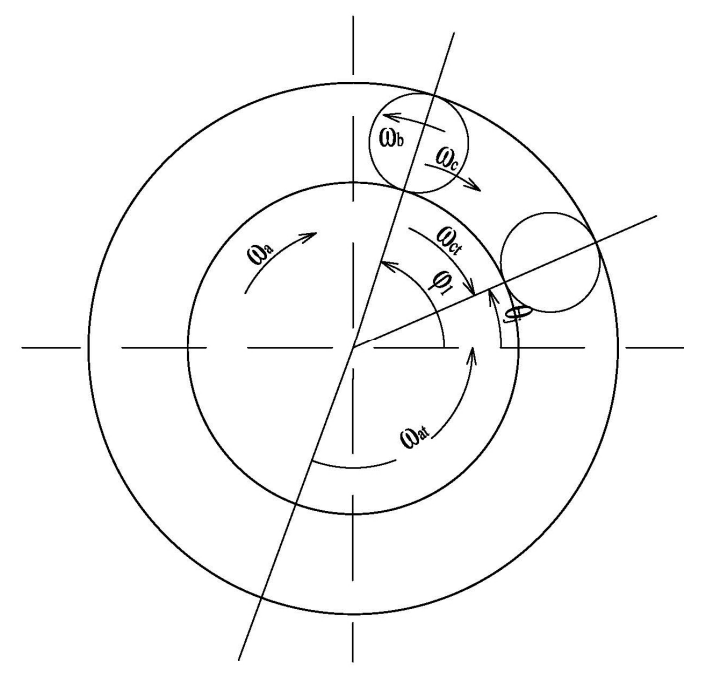

Fig. 1 The roller position

We took the intersection of the outer ring raceway and $\mathrm{x}$ axis as the reference origin. We ignored the contact deformation of the rolling element and the inner and outer ring raceway. We could get the radial displacement of the touch point of the outer ring raceway and rolling body from (1).

$$
\Delta \mathrm{r}_{j}=A \cos \left(\mathrm{n} \theta_{\mathrm{j}}+\varphi_{0}\right)
$$

Inner ring raceway waviness caused the touch point of outer ring raceway and rolling body $j$ had radial displacement. If we took the intersection of the bearing inner ring raceway before operation with $\mathrm{x}$ axis for corrugated reference origin, the radial displacement was

$$
\Delta \mathrm{r}_{j}=A \cos \left\lfloor\mathrm{n}\left(\theta_{\mathrm{j}}+\omega_{\alpha} t\right)+\varphi_{0}\right\rfloor
$$

Where: $\omega_{\alpha}$ is the rotation angular frequency of the bearing inner ring. Roller surface waviness caused the radial displacement of the outer ring raceway touch point. If we took the contact point of the bearing roller before operation and the outer ring raceway for corrugated reference origin, the radial displacement was

$$
\Delta \mathrm{r}_{j}=A_{\mathrm{j}} \cos \left(n \omega_{\mathrm{b}} t+\varphi_{0 j}\right)+A_{j} \cos \left[n\left(\omega_{b}+\pi\right)+\varphi_{0 j}\right]=2 A_{\mathrm{j}} \cos \frac{\mathrm{n} \pi}{2} \cos \left(n \omega_{b} t+\frac{n \pi}{2}+\varphi_{0 j}\right)
$$

Where: $\omega_{b}$ is roller rotation angular frequency; $\omega_{0 j}$ is the surface initial angle of the corrugated roller $j ; A_{j}$ is the surface ripple amplitude of the roller. When $n \neq 2 k(k=0,1,2, \ldots .),. \Delta r_{j}=0$. And when $n=2 k$ $(k=0,1,2 \ldots)$, there was radial displacement of the outer ring raceway contact point. These could be seen by the formula (6). The radial displacement was

$$
\Delta r_{j}=2 A_{\mathrm{j}} \cos \left(2 k \omega_{b} t+\varphi_{0 j}\right)_{n=2 k}
$$




\section{Outer ring vibration polar function and image analysis}

If the circular radius of the bearing outer ring raceway equilibrium position was $r_{0}$, at any time the location of the contact points of the outer ring raceway was $r_{j}$.

$$
r_{j}=r_{0}+\Delta r, j=1,2, \ldots, Z
$$

The outer ring raceway waviness was

$$
r_{j}=r_{0}+A \cos \left( \pm P \theta_{i}-k Z \Phi_{c} t+k Z \varphi_{1}+\varphi_{0}\right), \quad \theta_{j}=\frac{2 \pi}{Z}(j-1)+\varphi_{1}-\omega_{C} t, j=1,2, \ldots, Z
$$

For any time $j$ is the parameter coordinates. The $\theta_{j}$ is the polar angle. The $r_{j}$ is radius vector. (9) is the outer ring raceway position and form at any time. They were determined by the size of $P$. (9) was expressed as four groups of curve in Fig.2. The center circle was the balance position of the outer ring raceway.
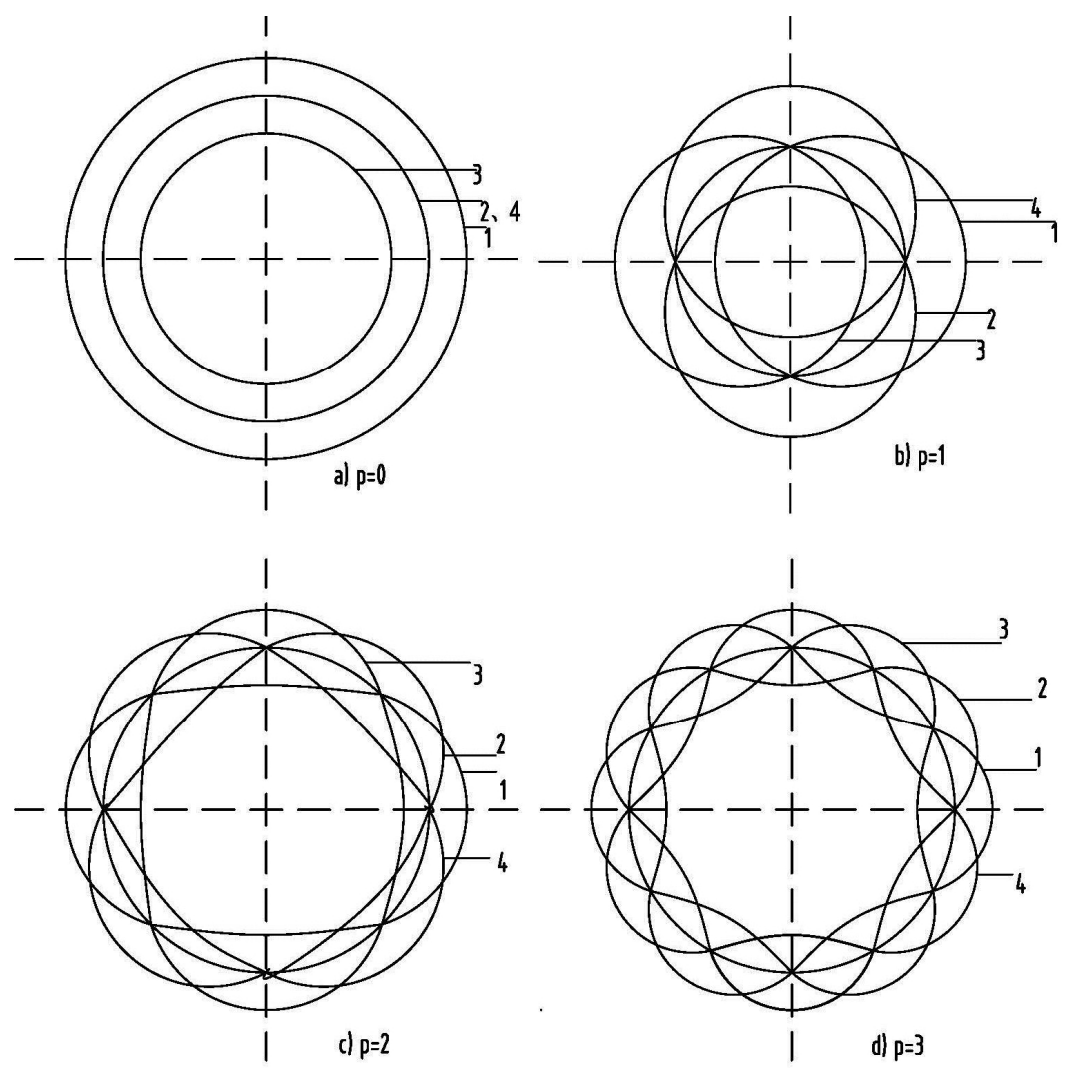

Fig.2 Vibration curve of bearing outer ring

When $P=0$ the radius of the graphics was crossfade. And the crossfade radius was on behalf of bearing radial clearance changes. Bearing inner ring and outer ring had axial relative movement. Mobile amount depended on the structure shape of the inner ring and outer ring raceway. When $n=k^{*} z$, the outer ring rigid vibration was produced. The frequency was $k^{*} z^{*} w_{c}$.

When $P=1$ graphics was a circle which had constant radius. And the circle was off-center circle. And the circle rotated around the center. When $n=k^{*} z \pm 1(P>1)$, any point on the outer radial had rigid vibration. The frequency was $k^{*} z^{*} w_{c}$.

When $P>1$ outer ring raceway had radial bending deformation. Raised portion was $P$, and which was the $(P-1)$ times of the radial bending. Deformation in the circumferential direction had rotation. Any point on the outer had radial displacement. So when $n=k^{*} z \pm 1(P>1)$, outer ring produced 
radial bending deformation which was the $(P-1)$ times and which was circumferential rotating. These caused the radial elastic vibration appeared on the outer.

Outer ring had radial or axial vibration. Influencing factors of the amplitude were as follows: ripple amplitude, bearing structure, contact deformation between rolling element and the inner or outer ring raceway, the outer radial stiffness. So we introduced a coefficient $K_{p}$. And we got the outer ring vibration caused by the outer ring raceway waviness.

$$
\Delta \mathrm{r}_{\theta}=K_{P} A \cos \left( \pm P \theta+k z \varphi_{1}+\varphi_{0}-k z \omega_{c} t\right)
$$

Where: $\theta$ is angle from any point on the outer ring of the position. In the general case $K_{0}$ and $K_{l}$ were bigger. With the increase of $P$ the $K_{p}$ was smaller and smaller. So the outer ring rigid vibration amplitude was greater than the amplitude of vibration caused by the elastic deformation. Inner ring raceway waviness, putting (5) into (8), we could get $r_{j}$ and $\theta_{j}$.

$$
r_{j}=r_{0}+A \cos \left\lfloor \pm P \theta_{j}+k Z\left(\omega_{\alpha}-\omega_{0}\right) t \pm P \omega_{\alpha} t+k Z \varphi_{1}+\varphi_{0}\right\rfloor \theta_{j}=\frac{2 \pi}{Z}(j-1)+\varphi_{1}-\omega_{c} t, j=1,2, \ldots, Z
$$

Compare (11) and (9), we found that in addition to the different vibration frequency, the direction and the type of the outer ring ripples caused by inner ring vibration were same with the outer ring corrugated case. The polar curve of (11) and (9) were exactly the same, as shown in figure 2 . The vibration of any point of the outer ring could be said to this

$$
\Delta \mathrm{r}_{\theta}=K_{P} A \cos \left[ \pm P \theta+K Z \varphi_{1}+\vartheta_{0}+K Z\left(\omega_{\alpha}-\omega_{0}\right) t \pm P \omega_{\alpha} t\right]
$$

The external waviness of body rolling, putting (7) into (8), we could get $r_{j}$.

$$
r_{j}=r_{0}+2 A_{j} \cos \left(2 k \omega_{b} t+\varphi_{0 j}\right)
$$

The vibration of the outer ring was more complex. Because there were differences of each roller surface ripple amplitude and phase angle.

$$
\begin{aligned}
& A_{j}=A, \varphi_{0 j}=\Phi \pm P \varphi_{j}=\phi \pm P\left(\theta_{j}+\omega_{c} t\right) \\
& r_{j}=r_{0}+2 A \cos \left( \pm P \theta_{j}+2 k \omega_{b} t \pm P \omega_{c} t+\Phi\right) \quad \theta_{j}=\frac{2 \pi}{Z}(j-1)+\varphi_{1}-\omega_{c} t, j=1,2, \ldots, Z
\end{aligned}
$$

Comparing (15) and (9) we could find that the direction and type of outer ring vibration were exactly the same in addition to the amplitude and frequencies were different. The polar curve of (15) and (11) were exactly the same, as shown in figure 2 . The vibration of any point of the outer ring could be said to this

$$
\Delta \mathrm{r}_{\theta}=K_{P} \bullet 2 A \cos \left\lfloor \pm P \theta_{j}+\left(2 k \omega_{b} \pm P \omega_{c}\right) t+\Phi\right\rfloor
$$

If you didn't meet (16), the same even external waviness of each corrugated roller would cause the outer ring the above three types of vibration at the same time. But the amplitude was less than $2 K_{p} * A * m, \quad A_{m}=\left(A_{1}+A_{2}+\ldots+A_{Z}\right) / Z$. 


\section{Conclusions}

The surface ripples of the inner and outer ring raceway and rolling body could be described using one or more harmonic function. When we studied the bearing outer ring vibration we took the contact point of the outer ring raceway and rolling element as the research object. We analyzed the polar function and image. The outer ring rigid vibration could also be analyzed using the least-square circle of the contact point of the outer ring raceway and rolling body. The analysis results were shown in table 1 . The surface ripples could cause rigid and elastic vibration of the bearing outer ring in the radial and axial. The amplitude related to the inner and outer ring raceway ripple amplitude. And it also related to the twice the amplitude of the external waviness of rolling body. Rigid vibration amplitude was greater than the elastic vibration amplitude. The direction, the type and the frequency of the vibration were associated with equivalent number. They were affected by the initial angle of the external waviness of rolling body as shown in Table 1.

Table 1 Relationship between $m * n$ and $\omega$

\begin{tabular}{cccc}
\hline part & $M^{*} n$ & $\omega$ & Vibration direction and type \\
\hline outer ring & $K Z \pm P$ & $K Z \omega_{c}$ & $P=0$ the axial rigidity of vibration \\
inner ring & $K Z \pm P$ & $K Z\left(\omega_{\alpha}-\omega_{c}\right) \pm P \omega_{\alpha}$ & $P=1$ the radial vibration rigidity \\
rolling body & $2 K$ & $2 K \omega_{b} \pm P \omega_{c}$ & $P>1$ the radial vibration caused by \\
the outer $(P-1)$ radial deformation
\end{tabular}

\section{References}

[1] Coperion Werner, Pfleiderer and Masterbatch. Plastics Additives \& Compounding. 3(2007) 36-39

[2] Strang G, Fix G, An Analysis of The Finite Methods, Prentice-Hall, New Jersey, 1973

[3] W. Strunk Jr., E.B. White, The Elements of Style, third ed., Macmillan, New York, 1979.

[4]Puccio F D, Gabiccini M and Guiggiani M. Mechanism and Machine Theory. 41(2006)382- 404.

[5] Ryken,Michael J,Vance,Judy M. Applying virtual reality techniques to the interactive stress anaslysis of a tractor life arm. Finite Element in Analysis and Design. 35(2000) 141-155.

[6] Rao S S. The Finite Element Method in Engineering. Pergamum Press, Oxford, 1982. 